Draft version March 29, 2018

Preprint typeset using $\mathrm{IAT}_{\mathrm{E}} \mathrm{X}$ style emulateapj v. 5/2/11

\title{
THE ECLIPSING BINARY CEPHEID OGLE-LMC-CEP-0227 IN THE LARGE MAGELLANIC CLOUD: PULSATION MODELLING OF LIGHT AND RADIAL VELOCITY CURVES
}

\author{
M. Marconi ${ }^{1}$, R. Molinaro ${ }^{1}$, G. Bono ${ }^{2,3}$, G. Pietrzyński ${ }^{4,5}$, W. Gieren ${ }^{5}$, B. Pilecki ${ }^{4,5}$, R. F. Stellingherf $^{6}$, D.

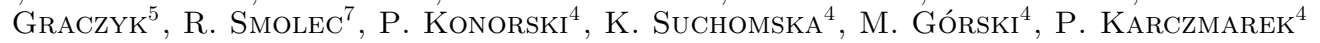 \\ Draft version March 29, 2018
}

\begin{abstract}
We performed a new and accurate fit of light and radial velocity curves of the Large Magellanic Cloud (LMC) Cepheid -OGLE-LMC-CEP-0227-belonging to a detached double-lined eclipsing binary system. We computed several sets of nonlinear, convective models covering a broad range in stellar mass, effective temperature and in chemical composition. The comparison between theory and observations indicates that current theoretical framework accounts for luminosity $-\mathrm{V}$ and $\mathrm{I}$ band-and radial velocity variations over the entire pulsation cycle. Predicted pulsation mass $-M=4.14 \pm 0.06 M_{\odot}-$ and mean effective temperature $-T_{e}=6100 \pm 50 \mathrm{~K}$ - do agree with observed estimates with an accuracy better than $1 \sigma$. The same outcome applies, on average, to the luminosity amplitudes and to the mean radius. We find that the best fit solution requires a chemical composition that is more metal-poor than typical LMC Cepheids $(\mathrm{Z}=0.004$ vs 0.008$)$ and slightly helium enhanced $(\mathrm{Y}=0.27$ vs 0.25$)$, but the sensitivity to He abundance is quite limited. Finally, the best fit model reddening $-E(V-I)=0.171 \pm 0.015 \mathrm{mag}-$ and the true distance modulus corrected for the barycenter of the $\mathrm{LMC}-\mu_{0, L M C}=18.50 \pm 0.02 \pm 0.10$ (syst) mag-, agree quite well with similar estimates in the recent literature.

Subject headings: stars: variables: Cepheids — binaries: eclipsing — Magellanic Clouds — stars: distances
\end{abstract}

\section{INTRODUCTION}

Classical Cepheids are among the most used primary distance indicators, currently at the base of an accurate extragalactic distance scale (Freedman et al. 2001; Saha et al. 2001; Freedman et al. 2011), thanks to their characteristic Period-Luminosity $(\mathrm{PL})$ relation. At the same time, these intermediate mass (typically from 3 to $13 M_{\odot}$ ) helium burning pulsating stars are used as tracers of relatively young $(\sim 10 \div 100 \mathrm{Myr})$ stellar populations. The physical basis of the PL relation is well known. By combining the period-density and the Stephan-Boltzmann relations, we obtain a PeriodLuminosity-Color-Mass (PLCM) relation. Stellar evolution predicts the occurrence of a Mass Luminosity (ML) relation for intermediate-mass He burning stars (Bono et al. 2000) and the PLCM can be easily converted into a Period-Luminosity-Color (PLC) relation holding for each individual Cepheid. The projection of the PLC

\footnotetext{
1 INAF-Osservatorio astronomico di Capodimonte, Via Moiariello 16, 80131 Napoli, Italy; marcella.marconi@oacn.inaf.it,molinaro@oacn.inaf.it

2 Dipartimento di Fisica - Università di Roma Tor Vergata, Via della Ricerca Scientifica 1, 00133 Roma, Italy; giuseppe.bono@roma2.infn.it

3 INAF-Osservatorio Astronomico di Roma, Via Frascati 33, 00040 Monte Porzio Catone, Italy

4 Warsaw University Observatory, Al. Ujazdowskie 4, 00-478, Warsaw, Poland; pietrzyn@astrouw.edu.pl, pilecki@astrouw.edu.pl, piokon@astrouw.edu.pl, ksenia@astrouw.edu.pl, mgorski@astrouw.edu.pl, pkarczmarek@astrouw.edu.pl

${ }^{5}$ Universidad de Concepción, Departamento de Astronomia, Casilla 160-C, Concepción, Chile; wgieren@astro-udec.cl, darek@astro-udec.cl

${ }^{6}$ Stellingwerf Consulting, 11033 Mathis Mtn Rd SE, 35803 Huntsville, AL USA; rfs@swcp.com

7 Nicolaus Copernicus Astronomical Centre, Bartycka 18, 00-716 Warszawa, Poland; smolec@camk.edu.pl
}

onto the $\mathrm{PL}$ plane gives the $\mathrm{PL}$ relation. On this basis, the investigation of Cepheid pulsation properties is also crucial for providing independent constraints on the ML relation.

In this context one of the most popular open problem is the so-called mass discrepancy problem suggested by Christy (1970) and Stobie (1969). The quoted authors noted that the Cepheid masses evaluated from the comparison of theoretical isochrones with observations were larger by almost a factor of two than the masses obtained from application of a period-massradius relation (Fricke et al. 1971). Even if a reduction of this discrepancy between evolutionary and pulsational masses was obtained by Moskalik et al. (1992) on the basis of updated radiative opacities, namely by the OPAL (Iglesias \& Rogers 1991) and the Opacity Project (Seaton et al. 1994) groups, several authors (Caputo et al.|2005; Natale et al.|2008; Bono et al.|2002; Keller \& Wood 2006; Evans et al. 2005; Brocato et al. 2003 ) noted tha the effect remained of the order of $10 \%$ $20 \%$ for both Galactic and Magellanic Cepheids.

A crucial input to this scenario was provided by the recent detection of two Cepheids in well detached, double-lined eclipsing binary systems in the LMC (Pietrzyński et al. 2010, 2011, OGLE-LMC-CEP-0227, OGLE-LMC-CEP-1812). The geometry and the precision of both photometric and spectroscopic data allowed the authors to measure the masses of these Cepheids with the unprecedented precision of $1 \%$, thus fixing a cornerstone in the solution of the mass discrepancy problem. Indeed, Cassisi \& Salaris (2011) were able to find evolutionary masses consistent with the dynamical estimate for OGLE-LMC-CEP-0227, on the basis of stellar evolution models that include a moderate amount of convective core overshooting, On the other hand, Neilson et al. 
(2011) found that both moderate convective core overshooting and pulsation-driven mass loss are required to achieve a solution of the mass discrepancy problem and Prada Moroni et al. (2012) adopt a new Bayesian approach to constrain the intrinsic stellar parameters of OGLE-LMC-CEP-0227, in particular its mass and age, by varying the efficiency of mass loss and core overshooting.

In this investigation we constrain the pulsation properties of OGLE-LMC-CEP-0227, by modelling its light and radial velocity curves with nonlinear convective models specifically computed for this purpose. In $\S 2$ we present the observational and theoretical framework, while in $\S 3$ we discuss the procedure adopted to fit light and velocity curves. In the same section, we also investigate the impact of metal and helium content on the best fit models. Conclusions and final remarks close the paper.

\section{OBSERVATIONAL AND THEORETICAL FRAMEWORK}

The observables we plan to use in the comparison between theory and observations are the $\mathrm{V}$ and I-band light curves (with the amplitudes $A_{V}, A_{I}$ ) and the radial velocity curve. Note that we define the light and the radial velocity curves observables even if they have been estimated by deblending the cumulative light and radial velocity curves of the binary system. To further constrain the projection factor, i.e. the parameter adopted to transform radial velocity into pulsation velocity, the radial velocity amplitude in our approach was assumed as a free parameter. The above observables together with the pulsation periods are available for a large number of classical Cepheids, in particular for those observed with one of the different flavors of the Baade-Wesselink method.

During the last few years it has been found that nonlinear, convective Cepheid models can play a crucial role in fitting the quoted observables (Bono et al. 1999, 2000, 2002; Keller \& Wood 2006). These models, once fixed the ML relation, provide very accurate fits of the above observables. On the basis of this theoretical framework and by assuming plausible values for the chemical composition (metal - $\mathrm{Z}$ - and helium $-\mathrm{Y}$ - abundances) and for the effective temperature very accurate fits have been provided not only for Galactic and Magellanic Cloud (MC) Cepheids (see e.g. Wood et al. 1997; Bono et al. 2002; Keller \& Wood 2006; Natale et al. 2008; Marconi et al. 2013), but also for other groups of radial variables (Marconi 2009). A further advantage of this nonlinear pulsation approach is that it provides independent estimates of the individual distance modulus and of the reddening (Bono et al. 2002; Marconi \& Clementini 2005; Marconi \& Degl'Innocenti 2007; Marconi et al. 2013).

Moreover, intrinsic parameters of radial variables based on pulsation observables can be compared with those based on evolutionary observables. However, this approach is partially hampered by the fact that pulsation models are envelope models, and therefore, they require the mass-luminosity relation predicted by evolutionary models. The binary LMC Cepheid OGLE-LMCCEP-0227 provides, for the first time, the opportunity to break this degeneracy. The unprecedented accuracy of the dynamical mass allows us to use a restricted range in mass values. Moreover, we also have for the same object a precise estimate of the mean effective temperature, this means that we can change the mean luminosity until predicted and observed period agree with each other. We still lack detailed information concerning the chemical composition, but for LMC Cepheids spectroscopic investigations are already available in the literature (Romaniello et al. 2008). The observed parameters of OGLE-LMC-CEP-0227 are listed in the first row of Table 18 .

\section{FIT OF LIGHT AND VELOCITY CURVES}

To perform an accurate fit of the pulsation observables $-\mathrm{V}$ and I-band light and radial velocity curves, we constructed a large set of pulsation models by adopting the typical chemical composition of $\mathrm{LMC}(\mathrm{Z}=0.008$, $\mathrm{Y}=0.25$ ) young stellar population (Luck et al. 1998; Romaniello et al. 2008; (Mucciarelli et al. 2011), the dynamical mass measured by Pietrzyński et al. (2010) and a broad range of effective temperatures with a step of 50 $\mathrm{K}$. The luminosity of individual models for each value of the effective temperature was changed in order to match the pulsation period9 (see Table 1). Fig. 1 shows the comparison between a subset of the computed models and the observations for OGLE-LMC-CEP-0227. Data plotted in this figure indicate that theory agrees quite well with observations.

However, to constrain on a quantitative basis the quality of the fit between the different sets of models and observations, we adopted a $\chi^{2}$ analysis. We phased the light and pulsational velocity curves of models in order to locate the maximum of the $\mathrm{V}$-band light curve at phase zero. Moreover, the best fit between theory and observations was computed by minimizing the following $\chi^{2}$ functions:

$$
\begin{gathered}
\chi_{P h}^{2}=\sum_{i=0}^{N_{\text {band }}} \sum_{j=1}^{N_{\text {points }}}\left[m_{j}^{i}-\left(M_{\text {mod }}^{i}\left(\phi_{j}^{i}+\delta \phi^{i}\right)+\delta M^{i}\right)\right]^{2}(1) \\
\chi_{V R}^{2}=\sum_{j=1}^{N_{\text {points }}}\left[v_{j}^{r a d}-\left(-\frac{1}{p} V_{\text {mod }}\left(\phi_{j}+\delta \phi_{V R}\right)+\gamma\right)\right]^{2}(2)
\end{gathered}
$$

From top to bottom they refer to light and radial velocity curves. Note that the index $i$ runs over the two photometric bands and $j$ over the number of measurements, $N_{\text {points }}=68$, 420, 135 for V, I and radial velocity, respectively. The predicted light and pulsational velocity curves are indicated with $M_{\text {mod }}$ and $V_{\text {mod }}$ and they have been evaluated at the same phase $-\phi_{j}-$ of the $\mathrm{j}^{\text {th }}$ measurement $-m_{j}, v_{j}^{r a d}-$ by fitting with a spline the predicted curves. The magnitude $\chi^{2}$ function is minimized with respect to shifts in $\delta M$ and to the possible shifts in phase $-\delta \phi-$ (typically ranging from 0.01 to 0.02 for the models listed in Table 1 ), while the velocity $\chi^{2}$ function is minimized with respect to the $\delta \phi$ (the same as for the photometry at the 0.01 level), the barycentric velocity $-\gamma-$ and the projection factor $p$, i.e. the parameter adopted to convert the observed radial velocity into pulsational velocity. The

\footnotetext{
8 The mean radius listed in column 11 is slightly different than the value given in Pietrzynski et al. (2010). The new estimate plus the projection factor $-\mathrm{p}-$ are based on the results by Pilecki et al. (2013, in preparation).

9 The exact match between observed and predicted period was fixed by using linear nonadiabatic pulsation models that provide the static envelope structure to the nonlinear hydrodynamical models (Bono et al. 1999). The period predicted by nonlinear models at limit cycle stability can differ from the linear one by a few percent.
} 


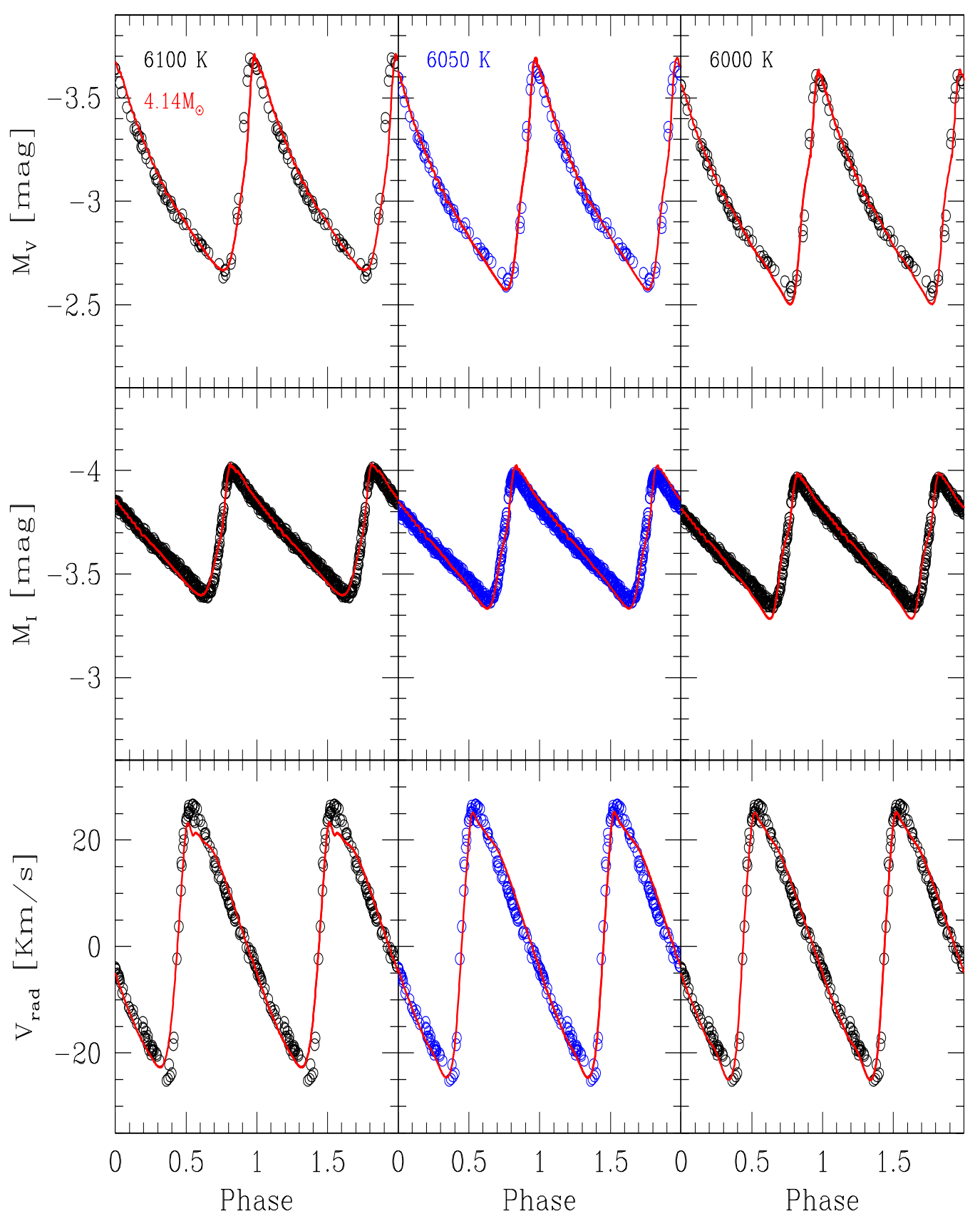

Fig. 1.- From top to bottom V- and I-band light curves and radial velocity curve. Open circles mark observations, while red lines display nonlinear pulsation models constructed at fixed stellar mass $\left(\mathrm{M}=4.14 M_{\odot}\right)$ and chemical composition $(\mathrm{Z}=0.008$, $\mathrm{Y}=0.25)$. From left to right the different panels show pulsation models constructed assuming different values of the mean effective temperature (labeled values). The luminosity of individual models was changed until predicted and observed period agreed with each other. The blue open circles mark the best fit solution, according to the total rms, for this set of models. The apparent magnitudes of the light curves were transformed into absolute magnitude using the true distance modulus and the reddening listed in Table 1. 
phase difference applied to light and radiative velocity curves are quite similar, and indeed we found that both the observed and the predicted phase lag, defined by using Fourier phases, between $\mathrm{V}$-band light and radial velocity curves is -0.2 . It is worth noting that the shifts in $\delta M$ provide the Cepheid true distance modulus and the reddening, while $\gamma$ gives the barycentric velocity of the system.

We neglected the models for which the $\chi^{2}$ analysis provided a shift in radial velocity $-\gamma-$ which are not consistent, within the errors, with the observed mean barycentric velocity (see Table 1). The rms for the different observables, given by the $\chi^{2}$ analysis, have been normalized to their pulsation amplitudes and summed in quadrature to obtain a total rms.

The best fit solution, for each set of models, satisfy the above requirements concerning the shifts and attains the smallest total rms. The best fit model is plotted with open blue circles in Fig. 1 and the intrinsic parameters are listed in Tab1 (see discussion below).

By applying the $\chi^{2}$ analysis to the models computed at fixed chemical composition $(\mathrm{Z}=0.008, \mathrm{Y}=0.25)$ and stellar mass $\left(M=4.14 M_{\odot}\right)$, we find that the effective temperature of the best fit model $-T_{e}=6050 \pm 50 \mathrm{~K}$ (see Fig. 1)- agrees quite well with the empirical estimate $\left(T_{e}=5900 \pm 250 \mathrm{~K}\right)$. The same outcome applies to the other observables, since the agreement between theory and observations is better than $1 \sigma$ for the mean radius and the V-band amplitude, and of the order of $2 \sigma$ for the I-band. Note that the uncertainties on the intrinsic parameters of the best fit model were estimated according to the step in mass value and in effective temperature of the different sets of models.

To further improve the accuracy of the intrinsic parameters, we computed a new set of models at fixed chemical composition and effective temperature $\left(T_{e}=6050 \mathrm{~K}\right)$. The new models were constructed assuming a step in mass of $\sim 0.06 M_{\odot}$ and once again the luminosity was changed to fit the observed period. The $\chi^{2}$ analysis indicates that the best fit is given by a model with a stellar mass of $4.08 M_{\odot}$ and a luminosity level $\log L / L_{\odot}=3.13$ dex. Data plotted in Fig. 2, where a subset of the computed models is shown, display that theory and observation agree quite well with each other.

\subsection{Dependence on chemical composition}

In order to constrain the dependence of the best fit solution on the adopted chemical composition, we computed additional pulsation models by varying either the metallicity or the helium content. In particular, we computed new sets of models at fixed helium content $-\mathrm{Y}=0.25$ - and three different metal abundances: $\mathrm{Z}=0.006, \mathrm{Z}=0.004$ and $\mathrm{Z}=0.002$. For each set of models, we performed the same fit to the observed curves both at fixed mass and at fixed effective temperature, following the same approach we adopted for the models at canonical chemical composition $(\mathrm{Z}=0.008)$. The best fit solutions for $\mathrm{Z}$ ranging from 0.004 to 0.008 are plotted in Fig. 3 and the corresponding stellar parameters for all the metallicities are listed in Table 1 .

The total rms of the best fit models for the four different metallicities, indicate that the global best fit is given by the model with $\mathrm{Z}=0.004, \mathrm{Y}=0.25, \mathrm{M}=4.26 \pm 0.06$ $M_{\odot}, \log L / L_{\odot}=3.15 \pm 0.02$ and an effective temperature of $6050 \pm 50 \mathrm{~K}$, again in good agreement with the results by Pietrzyński et al. (2010) (see also Table 1) 10 . Current analysis further confirms the sensitivity of pulsation models to the metallicity, and in turn the opportunity to constrain this pa-

10 The match of the model at $\mathrm{Z}=0.002$ with the data predicts a barycentric velocity $-\gamma-$ at odds with the mean value given by Pietrzyński et al. (2010) and for this reason it was not taken into account (see $\S 3$ ). However, it was kept in the analysis only for the sake of completeness. rameter with the comparison between predicted and observed light and radial velocity variations (Natale et al. 2008).

To further constrain the impact that chemical composition has on the comparison between predicted and empirical observables, we also computed new sets of pulsation models at fixed metallicity $-\mathrm{Z}=0.004-$ and three enhanced helium contents, namely $\mathrm{Y}=0.26,0.27,0.28$. The dependence of the best fit solution on the helium content is shown in Fig. 4. Data plotted in this figure and predicted observables listed in Table 1 indicate that the best fit solutions are those with He enhanced ( $\mathrm{Y}=0.27, \mathrm{Y}=0.28)$ composition. However, the rms values clearly show that pulsation observables are less sensitive to changes in He than in metal content. On the basis of this evidence and the fact that the set of models with the highest He content $(Y=0.28)$ give barycentric velocity $\gamma$ - slightly larger than the value given by Pietrzvński et al. (2010), we assume that our global best fit model is the one with $\mathrm{Z}=0.004$ and $\mathrm{Y}=0.27$.

\section{CONCLUSIONS AND FINAL REMARKS}

We computed new sets of nonlinear convective pulsation models in order to match the observed V- and I-band light curve, the radial velocity curve and the radius curve of the LMC Cepheid OGLE-LMC-CEP-0227. This variable belongs to a detached, double-lined eclipsing binary system, and for the first time its dynamical mass was estimated with an accuracy better than 1\% Pietrzyński et al. (2010). By adopting the canonical chemical composition for LMC Cepheids $(\mathrm{Z}=0.008, \mathrm{Y}=0.25)$, the best fit model gives a value of the Cepheid mass $-M=4.08 \pm 0.06 M_{\odot}-$ and of the effective temperature $-T_{e}=6050 \pm 50 \mathrm{~K}$ - that agree quite well with the observed values. This outcome also applies to the mean radius (the difference is smaller than $1 \sigma$ ) and to the pulsation amplitudes (the difference is smaller than $2 \sigma)$. The true distance modulus corresponding to this solution is $\mu_{0}=18.44 \pm 0.02$ where the small uncertainty is the only contribution of the model fitting technique. By adding a conservative systematic error of $0.1 \mathrm{mag}$ to account for several residual uncertainties introduced by the adopted turbulent convective model, the static model atmosphere, the input physics, together with observational errors, we found that current distance is in remarkable agreement with several independent estimates of the LMC distance in the recent literature (Bono et al. 2002; Marconi \& Clementini 2005; Keller \& Wood 2006; Testa et al.|2007; |Molinaro et al. 2012; Marconi et al. 2013; (Pietrzyński et al. 2013). To provide a firm estimate of the LMC distance we evaluated the geomtrical correction by adopting the model by van der Marel et al. (2002) and we found that it is $0.062 \mathrm{mag}$. Therefore, current distance modulus once corrected for the barycenter of the LMC becomes $\mu_{0, L M C}=18.50 \pm 0.02 \mathrm{mag}, \quad(d=50.1 \pm 0.5$ $\mathrm{kpc}$ ) that is in excellent agreement with the very precise distance recently provided by Pietrzyński et al. (2013) using eight long-period, late-type double eclipsing binary systems $(18.49 \pm 0.05 \mathrm{mag})$. The same agreement applies to the distance modulus provided by Prada Moroni et al. (2012) using evolutionary models (18.53 $\pm 0.02 \mathrm{mag})$.

However, we still lack an accurate measurement of the iron abundance of this Cepheid. Therefore, we also investigated possible variations of the chemical compositions. We found that the best fit model providing pulsation observables that agree quite well with observations and an even smaller rms is the model with $\mathrm{Z}=0.004, \mathrm{Y}=0.27, M=4.14 \pm 0.06 M_{\odot}$, $\log L / L_{\odot}=3.16 \pm 0.02 \operatorname{dex}, T_{e}=6100 \pm 50 \mathrm{~K}$ and $R=34.3 \pm$ $0.2 R_{\odot}$. This model provides a distance modulus corrected for the barycenter of the LMC of $\mu_{0, L M C}=18.50 \pm 0.02 \mathrm{mag}$.

Current pulsation mass estimate agrees quite well with the dynamical mass measurements. A similar agreement has also been found concerning the evolutionary masses suggesting 
TABLE 1

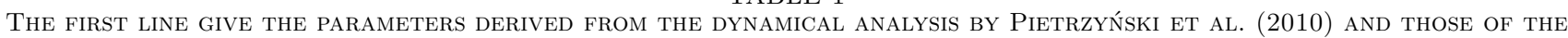
OBSERVED PHOTOMETRIC AND RADIAL VELOCITY CURVES, WHILE THE BEST FIT MODEL PARAMETERS ARE LISTED IN THE FOLLOWING SEVEN lines of the table: Metallicity (Column 1), Helium content (Column 2), Mass in solar units (COlumn 3), Luminosity (COlumn 4), Effective Temperature (COlumn 5), Period (Derived from the linear and the nonlinear analysis) IN Days (COlumn 6 ),

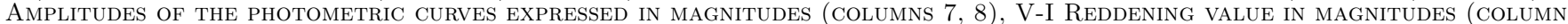
9), dereddened Distance Modulus in magnitudes (column 10), the mean Radius (column 11), Projection Factor (column 12), Barycentric Velocity in KM/s (COLUmn 13) and Total RMS (COLUmN 14).

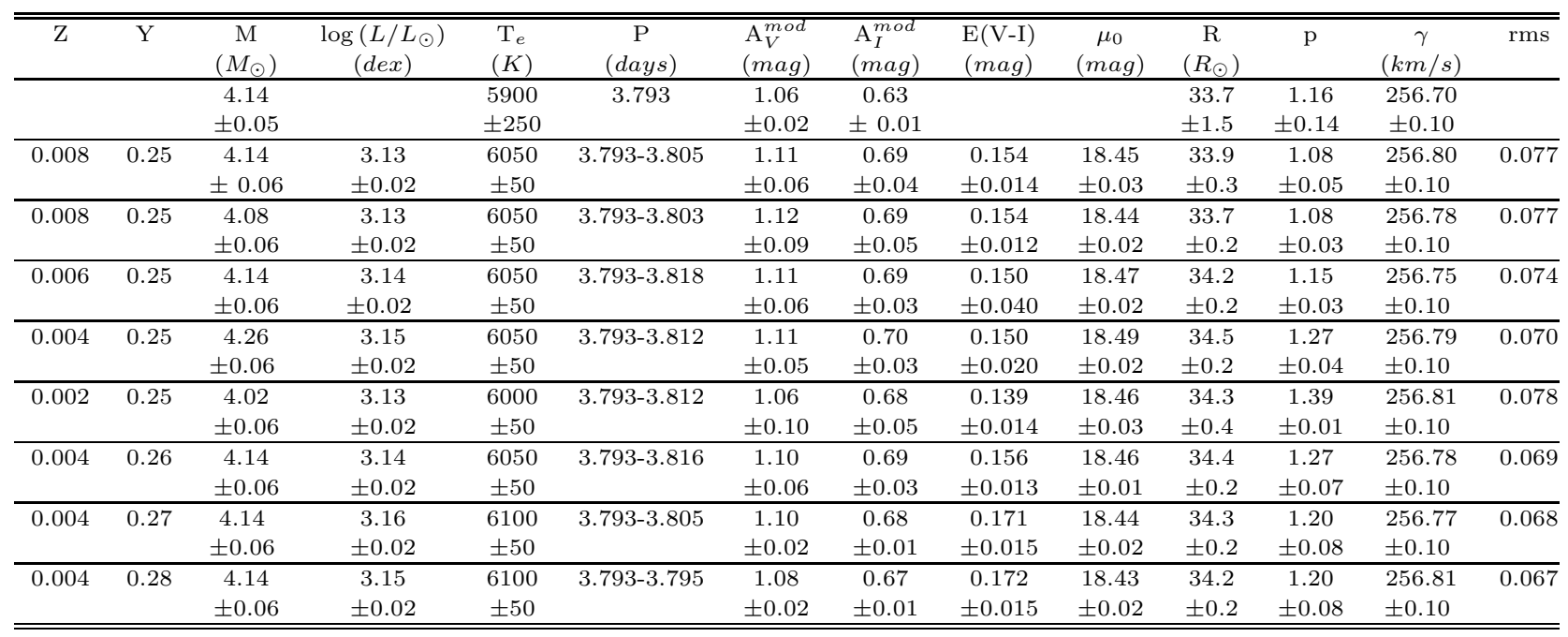

either an increase in the efficiency of convective core overshooting during hydrogen burning phases (Cassisi \& Salaris 2011; Prada Moroni et al. 2012) and a pulsation driven mass loss inside the Cepheid instability strip (Neilson et al. 2011). Current pulsation and evolutionary results are hampered by the fact that they are only based on a single object and are affected by degeneracy among the different input parameters. The use of independent solid observables (period derivative, CNO abundances) will provide firm constraints on the actual evolutionary and pulsation status of the system (Neilson et al. 2012; Matthews et al. 2012).

The $p$-factor given by our best fit solution $-1.20 \pm 0.08$ is consistent with the result obtained for the prototype $\delta$ Cephei by Mérand et al. (2005) and with the value suggested by Groenewegen (2013) by adopting Galactic Cepheids with individual distances based on trigonometric parallaxes. The current estimate is also in very good agreement with the new binary best fit solution provided by Pietrzynski et al. (2013) and confirms the value predicted by Nardetto et al. (2004) on the basis of Cepheid hydrodynamical models. However, the $p$ factor we found is smaller than the values recently determined for short-period Cepheids in the LMC by Storm et al. (2011). The $p$-factor and its possible dependence on the pulsation period is still lively debated (Gieren et al. 2005; Groenewegen 2013; Ngeow et al. 2012) and we need to apply the same analysis to several Cepheids before we can rich firm conclusions.

The reddening estimate is also in very good agreement with recent evaluations based on reddening maps (Schlegel et al. 1998) and on evolutionary models (Prada Moroni et al. 2012). The estimated luminosity level is in remarkable agreement not only with the estimate of the binary modelling Pietrzyński et al. (2010), but also with evolutionary prescriptions based on models accounting for mild convective core overshooting during central hydrogen burning and canonical mass loss rates (see figure 3 in Prada Moroni et al. 2012). This evidence indicates a very promising agreement between pulsation and evolutionary observables. Finally, it is worth mentioning that the nonlinear pulsation approach can provide firm constraints not only on the pulsation observables -at a few percent level- but also on the metal abundance. However, their sensitivity to the helium content is quite limited.

We acknowledge financial support from PRIN INAF 2011 (P.I. M. Marconi) and from PRIN MIUR 2011 (P.I. F. Matteucci). G.B. thanks ESO for support as science visitor. WG and GP thank for support from the BASAL Centro de Astrofisica y Tecnologias Afines (CATA) PFB-06/2007. Support from Foundation for Polish Science (program TEAM) and the Polish National Science Centre (program MAESTRO) is also acknowledged. RS is supported by Polish NCN (grant DEC2012/05/B/ST9/03932). We dedicate this manuscript to the memory of R. Buchler. His suggestions and ideas in the field of stellar pulsation will be greatly missed. 


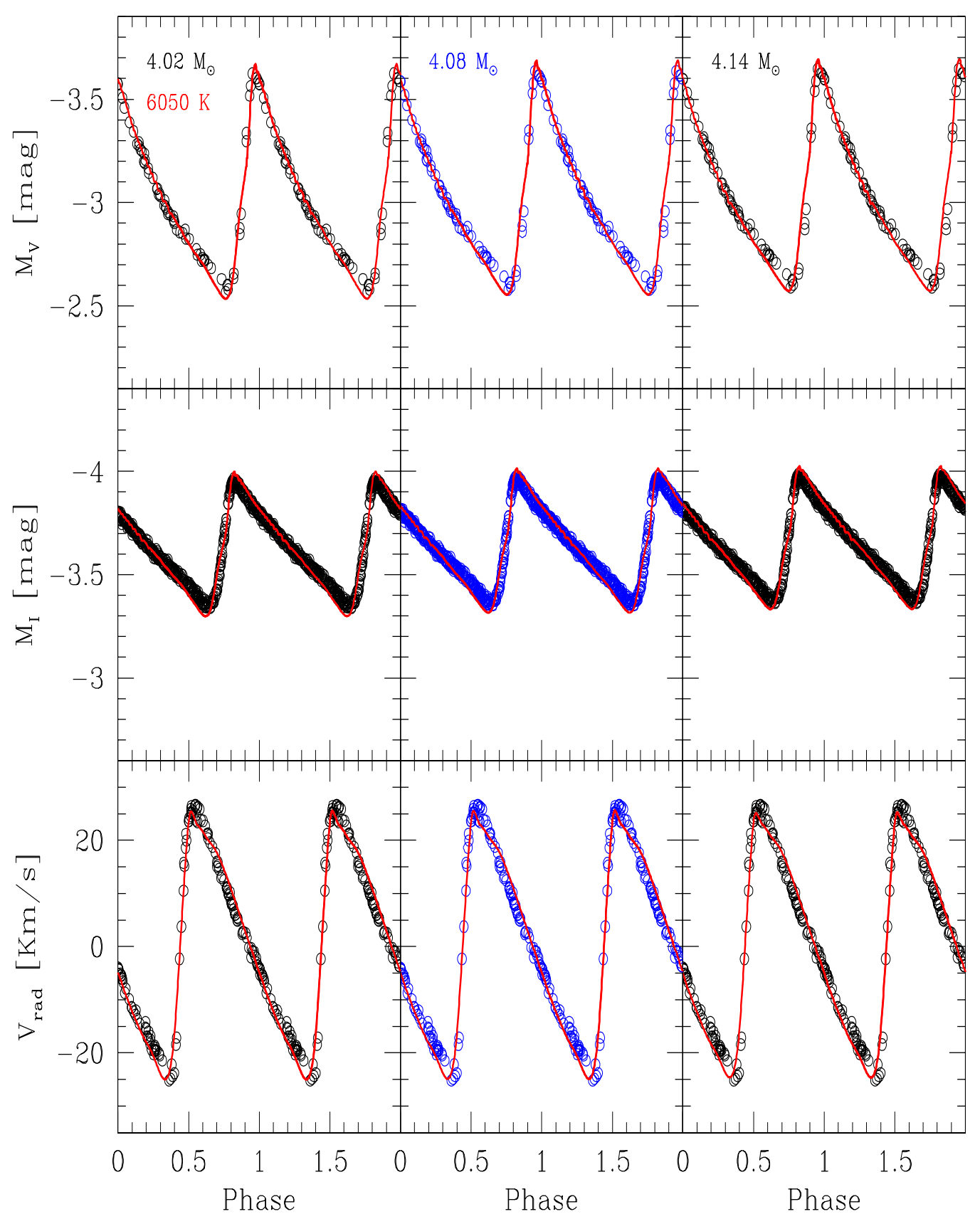

FIG. 2. - Same as Fig. 1, but the comparison between theory and observations was performed at fixed chemical composition $(\mathrm{Z}=0.008$, $\mathrm{Y}=0.25)$ and effective temperature $\left(T_{e}=6050 \mathrm{~K}\right)$. From left to right the different panels display pulsation models constructed by assuming different values of the stellar mass (see labeled values). The blue circles show the best fit solution for this set of models.

\section{REFERENCES}

Bono, G., Marconi, M., \& Stellingwerf, R. F. 1999, ApJS, 122, 167

Bono, G., Caputo, F., Cassisi, S., et al. 2000, ApJ, 543, 955

Bono, G., Marconi, M., \& Stellingwerf, R. F. 2000, A\&A, 360, 245

Bono, G., Castellani, V., \& Marconi, M. 2002, ApJ, 565, L83

Bono, G., Groenewegen, M. A. T., Marconi, M., \& Caputo, F. 2002, ApJ, 574, L33

Bono, G., Caputo, F., Fiorentino, G., Marconi, M., \& Musella, I. 2008, ApJ, 684, 102
Bono, G., Caputo, F., Marconi, M., \& Musella, I. 2010, ApJ, 715, 277

Brocato, E., Castellani, V., Di Carlo, E., Raimondo, G., \&

Walker, A. R. 2003, AJ, 125, 3111

Caputo, F., Bono, G., Fiorentino, G., Marconi, M., \& Musella, I. 2005, ApJ, 629, 1021

Cassisi, S., \& Salaris, M. 2011, ApJ, 728, L43

Christy, R. F. 1970, JRASC, 64, 8 


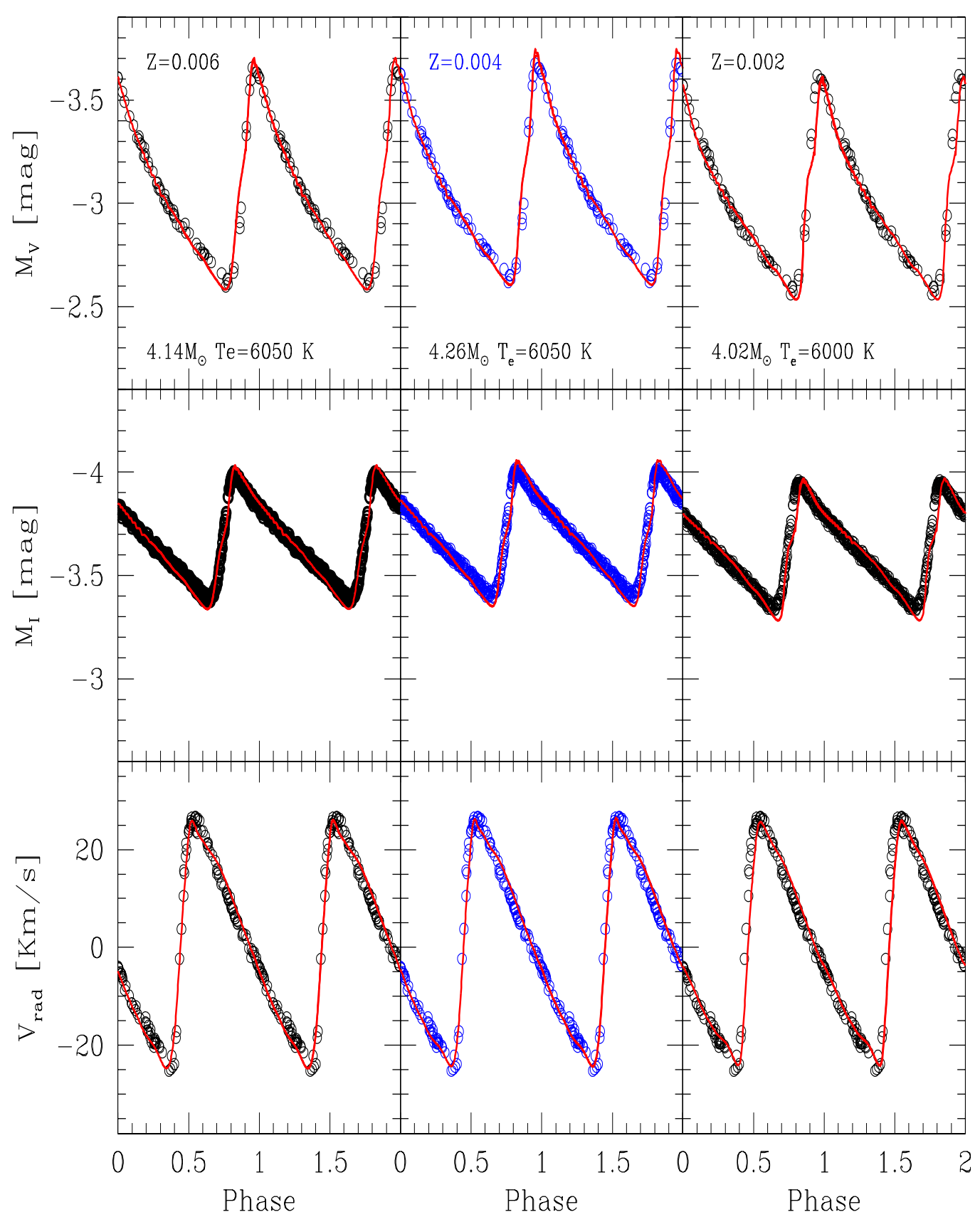

FIG. 3.- Same as Fig. 1, but for pulsation models constructed by assuming the same helium content $(\mathrm{Y}=0.25)$ and different values of the metal abundance (see labeled values). The stellar mass and the effective temperature of the best fit solution, for each set of models, are also labeled. Blue circles display the best fit solution of the current set of models.

Evans, N. R., Carpenter, K. G., Robinson, R., Kienzle, F., \& Dekas, A. E. 2005, AJ, 130, 789

Freedman, W. L., Madore, B. F., Gibson, B. K., et al. 2001, ApJ, 553,47

Freedman, W. L., Madore, B. F., Scowcroft, V., et al. 2011, AJ, 142,192

Fricke, K., Stobie, R. S., \& Strittmatter, P. A. 1971, MNRAS, 154, 23

Gieren, W., Storm, J., Barnes, T. G., III, et al. 2005, ApJ, 627, 224

Groenewegen, M. A. T. 2013, A\&A, 550, A70
Iglesias, C. A., \& Rogers, F. J. 1991, ApJ, 371, 408 Keller, S. C., \& Wood, P. R. 2006, ApJ, 642, 834

Luck, R. E., Moffett, T. J., Barnes, T. G., III, \& Gieren, W. P. 1998, AJ, 115, 605

Marconi, M. 2009, Mem. Soc. Astron. Italiana, 80, 141

Marconi, M., \& Clementini, G. 2005, AJ, 129, 2257

Marconi, M., \& Degl'Innocenti, S. 2007, A\&A, 474, 557

Marconi, M., Musella, I., Fiorentino, G., et al. 2010, ApJ, 713, 615

Marconi, M., Molinaro, R., Ripepi, V. et al. 2013, MNRAS, 428, 2185 


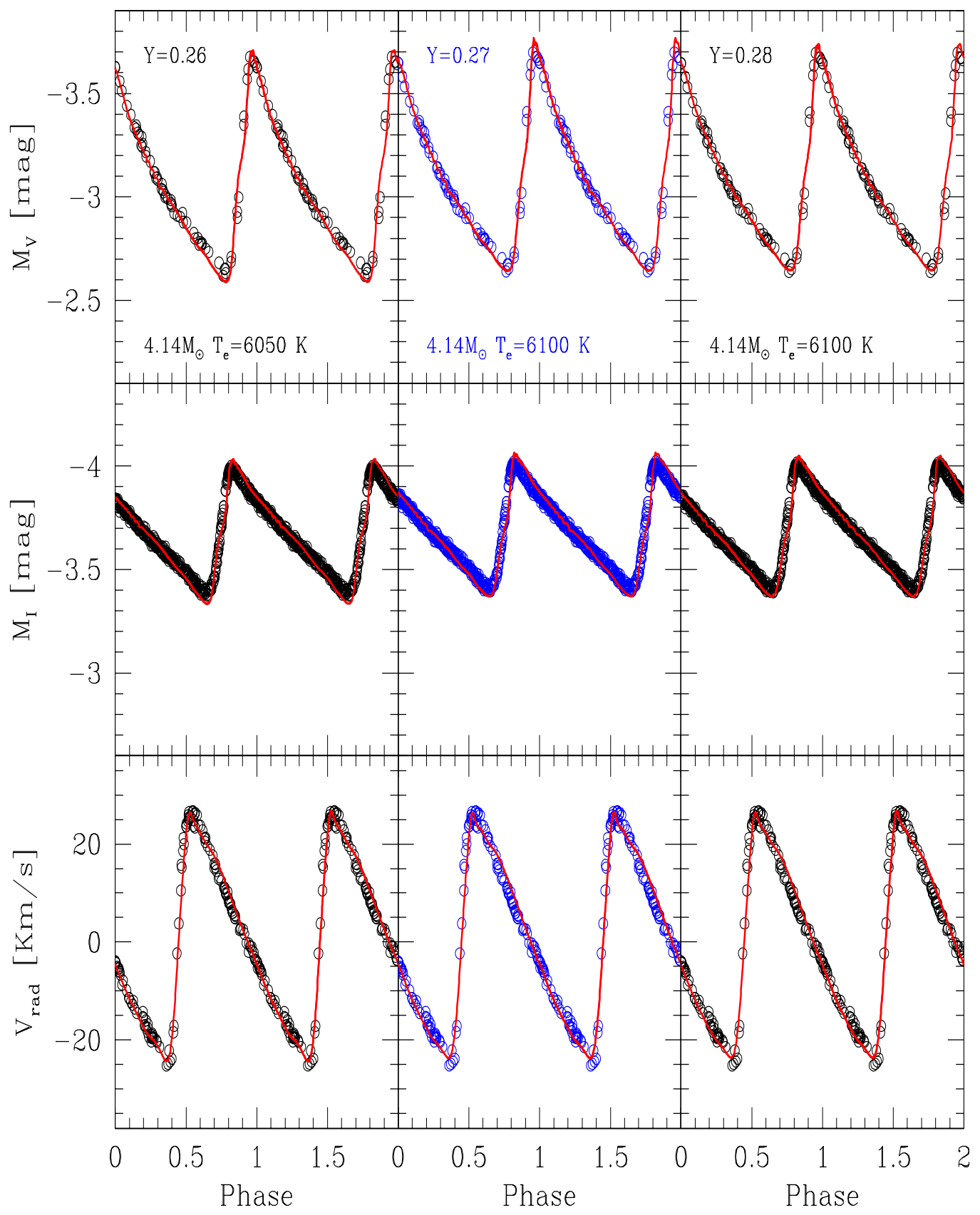

FIG. 4. - Same as Fig. 3, but for pulsation models constructed by assuming the same metal content $(\mathrm{Z}=0.004)$ and different helium abundances (see labeled values). The helium enhanced $(Y>0.25)$ pulsation models cover a broad range in stellar mass and in effective temperature. The stellar mass and the effective temperature of the best fit solution, for each set of models, are also labeled. Blue circles display the global best fit solution. 
Matthews, L. D., Marengo, M., Evans, N. R., \& Bono, G. 2012, ApJ, 744, 53

Mérand, A., Kervella, P., Coudé du Foresto, V., et al. 2005, A\&A, 438, L9

Molinaro, R., et al., 2012, ApJ, 748, 69

Moskalik, P., Buchler, J. R., \& Marom, A. 1992, ApJ, 385, 685

Mucciarelli, A., Cristallo, S., Brocato, E., et al. 2011, MNRAS, 413,837

Nardetto, N., Fokin, A., Mourard, D., et al. 2004, A\&A, 428, 131

Natale, G., Marconi, M., \& Bono, G. 2008, ApJ, 674, L93

Neilson, H. R., Cantiello, M., \& Langer, N. 2011, A\&A, 529, L9

Neilson, H. R., Langer, N., Engle, S. G., Guinan, E., \& Izzard, R. 2012, ApJ, 760, L18

Ngeow, C.-C., Neilson, H. R., Nardetto, N., \& Marengo, M. 2012, A\&A, 543, A55

Pietrzyński, G., Thompson, I. B., Gieren, W., et al. 2010, Nature, 468, 542

Pietrzyński, G., Thompson, I. B., Graczyk, D., et al. 2011, ApJ, 742, L20
Pietrzyński, G., Graczyk, D., Gieren, W., et al. 2013, Nature, 495, 76

Prada Moroni, P. G., Gennaro, M., Bono, G., et al. 2012, ApJ, 749, 108

Romaniello, M., Primas, F., Mottini, M., et al. 2008, A\&A, 488, 731

Saha, A., Sandage, A., Tammann, G. A., et al. 2001, ApJ, 562, 314

Schlegel, D. J., Finkbeiner, D. P., \& Davis, M. 1998, ApJ, 500, 525

Seaton, M. J., Yan, Y., Mihalas, D., \& Pradhan, A. K. 1994, MNRAS, 266, 805

Stobie, R. S. 1969, MNRAS, 144, 511

Storm, J., Gieren, W., Fouqué, P., et al. 2011, A\&A, 534, A95

Testa, V., et al., 2007, A\&A, 462, 599

van der Marel, R. P., Alves, D. R., Hardy, E., \& Suntzeff, N. B. 2002, AJ, 124, 2639

Wood, P. R., Arnold, A., \& Sebo, K. M. 1997, ApJ, 485, L25 